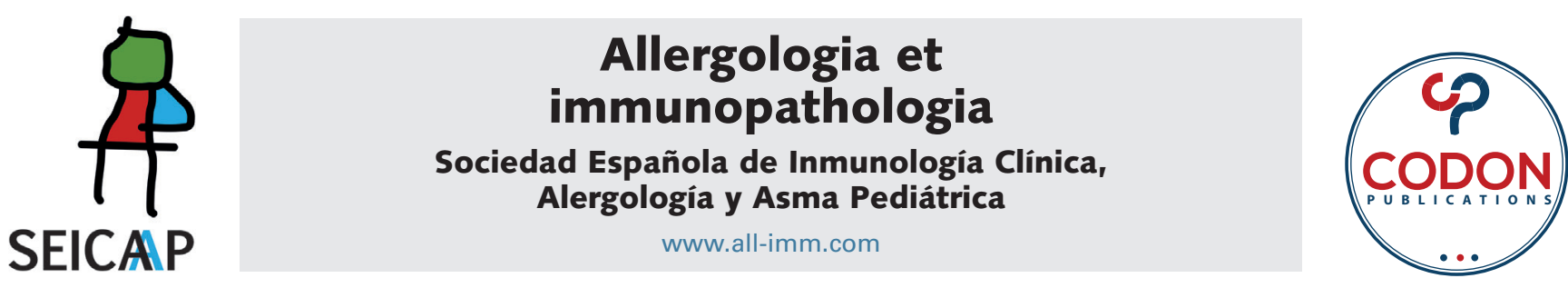

RESEARCH ARTICLE

\title{
METTL3 promotes inflammation and cell apoptosis in a pediatric pneumonia model by regulating EZH2
}

\author{
Ying Yang ${ }^{\mathrm{a}}$, Xiaoqing Yanga*, Yi Wu ${ }^{\mathrm{b}}$, Meijiao $\mathrm{Fu}^{\mathrm{a}}$
}

aDepartment of Pediatrics, Women and Children's Hospital, School of Medicine, Xiamen University, Xiamen, Fujian China ${ }^{b}$ Department of Pediatrics, Maternal and Child Health Hospital of Guangxi Zhuang Autonomous Region, Nanning, Guangxi Zhuang Autonomous Region 530000, China

Received 14 May 2021; Accepted 25 June 2021

Available online 1 September 2021

\section{KEYWORDS \\ EZH2; \\ JAK2; \\ METTL3; \\ pediatric pneumonia; \\ STAT}

\begin{abstract}
Background: In recent times, the medical science has developed by leaps and bounds, however, the molecular mechanism of pediatric pneumonia is still unclear. Although prior researches have shown that methyltransferase-like 3 (METTL3) is up-regulated in a variety of inflammatory diseases, its role and mechanism has been rarely studied in pediatric pneumonia, and need to be defined elaborately.

Objective: In this study, the related molecular mechanism of METTL3 on inflammation and cell apoptosis in a pediatric pneumonia was investigated.

Materials and methods: Quantitative real-time polymerase chain reaction (qPCR) and western blot assays were employed to examine the mRNA and protein expression level of METTL3 and EZH2 in peripheral blood monocytes from pediatric pneumonia patients or cell model (WI-38). Then, qPCR and ELISA assay were applied to verify the inflammatory response in LPS-treated WI-38 cell lines after knockdown of METTL3. Besides, MTT cell viability assays, flow cytometry, and western blot assays were applied to examine the cell viability and cell apoptosis of LPS-treated WI-38 cell after knockdown of METTL3. Further, the western blot assays were employed to examine the protein expression levels of p-JAK2, JAK2, p-STAT3, STAT3, and EZH2 in LPS-treated WI-38 cell after knockdown of METTL3. Finally, ELISA and western blot were applied to verify the inflammatory response and cell apoptosis of LPS-treated WI-38 cell after knockdown of METTL3 and overexpression of EZH2.

Results: In this study, the results showed that METTL3 and EZH2 were highly expressed in pediatric pneumonia patients and cell models (WI-38), respectively. Besides, downregulation of METTL3 inhibited LPS-induced inflammatory response and cell apoptosis. Then, the fact that METTL3 regulates the JAK2/STAT3 signaling pathway through EZH was proved. Furthermore, downregulation of METTL3 inhibits inflammation and apoptosis through EZH2.
\end{abstract}

*Corresponding author: Xiaoqing Yang, Department of Pediatrics, Women and Children's Hospital, School of Medicine, Xiamen University, No. 10, Zhenhai Road, Zhonghua Street, Siming District, Xiamen, Fujian 361003, China. Email address: yangxiaoqing0423@163.com 
Conclusion: This study found that METTL3 promotes inflammation and cell apoptosis in a pediatric pneumonia model by regulating EZH2.

(c) 2021 Codon Publications. Published by Codon Publications.

\section{Introduction}

Pneumonia is one of the major infectious diseases, and its global mortality and morbidity are at a leading level, especially in children. Around 1.1-1.4 million children die of pneumonia annually. The high incidence of pneumonia in children can lead to serious complications and adverse reactions, and even death. ${ }^{1}$ Therefore, it is necessary to study the potential pathogenesis of pneumonia in children and identify effective therapeutic targets. ${ }^{2}$ Human lung fibroblasts WI-38 treated with lipopolysaccharide (LPS) to construct a pneumonic cell model is often used to study pediatric lung disease. Activating proinflammatory factor, in turn, can activate a variety of intracellular signal transduction pathways, including Janus kinase 2 (JAK2)-signal transduction and transcriptional activator 3 (STAT3) pathway, which is a proinflammatory and apoptotic pathway. JAK2 and its downstream factor STAT3 (the JAK2-STAT3 signaling pathway) are triggered by ligand, or LPS stimulation, if activated STAT3 is translocated into the nucleus to regulate target gene expression, ${ }^{3,4}$ and this JAK2-STAT3 activation induces pneumonia. ${ }^{5}$

Methyltransferase-like 3 (METTL3) is an RNA N-6methyladenosine (M6A) methyltransferase, which is essential for the modification of M6A mRNA. As a key enzyme of $M 6$, it is involved in immune and inflammatory regulation. Studies have shown that METTL3 is upregulated in a variety of inflammatory diseases, including rheumatoid arthritis ${ }^{6}$ and pulpitis. ${ }^{7}$ Knockdown of METTL3 reduces inflammation and apoptosis of chondrocytes. ${ }^{8}$ Loss of METTL3 further inactivates the JAK2-STAT3 pathway by interfering JAK2 and SOCS3 expression. ${ }^{9}$ Studies have shown that METTL3 can bind to EZH2 mRNA, and M6A modification was confirmed on EZH2 mRNA. After interference with METTL3, the expression of EZH2 protein in SUNE-1 cells decreases significantly. ${ }^{10}$ However, the role of METTL3 in pediatric pneumonia has remained unclear.

The enhancer of $\mathrm{EZH} 2$ is a histone lysine $\mathrm{N}$-methyltransferase, a vital component of multicomb inhibition complex 2. ${ }^{11}$ Studies have shown that EZH2 is upregulated in some inflammatory diseases, for example, in pulpitis. Inhibition of $\mathrm{EZH} 2$ can suppress the expression of proinflammatory factors, such as interleukin 6 (IL-6) and interleukin 8 (IL-8) cytokines, and weaken inflammatory response. ${ }^{12} \mathrm{EZH} 2$ is upregulated in nonalcoholic steatohepatitis, and treatment with EZH2 inhibitors reduces the expression of serum tumor necrosis factor- $\alpha$ (TNF- $\alpha){ }^{13}$ EZH2 may promote the proliferation and migration of bladder cancer through the JAK2-STAT3 pathway. ${ }^{14}$

Therefore, the role of METTL3 and its related mechanisms in pediatric pneumonia were studied in this study to provide reference for the treatment of the disease.

\section{Materials and methods}

\section{Tissue sample}

A total of 50 peripheral blood samples of pediatric pneumonia patients were randomly collected at the Women and Children's Hospital, School of Medicine, Xiamen University. Informed written consent was obtained from all patients before collection of samples. Study protocols were approved by the Ethics Committee of the Women and Children's Hospital, School of Medicine, Xiamen University (approval No. 2016-24, dated 09 March, 2016) and conducted according to the principles of the Declaration of Helsinki. ${ }^{15}$ The detail information were showed in the table 1.

\section{Cell culture}

The human embryonic lung cells (WI-38) were bought from the Chinese Academy of Sciences (Shanghai, China). The cells were cultured in Dulbecco's Modified Eagle Medium (DMEM; Gibco, Carlsbad, CA, USA) containing 10\% fetal bovine serum (Gibco) and $1 \%$ penicillin/streptomycin (Gibco) in a humidified $5 \% \mathrm{CO}_{2}$ incubator at $37^{\circ} \mathrm{C}$.

\section{Real-time quantitative polymerase chain reaction (RT-qPCR)}

Cells were collected and total RNA was extracted by using Trizol reagent (Invitrogen, CA, USA) as directed in the product manual. The ChamQ ${ }^{T M}$ SYBR $^{\circledR}$ qPCR Master Mix (Vazyme,

Table 1 Primers for METTL3, EZH2, IL-6, MCP-1, TNF- $\alpha$, and reference genes.

\begin{tabular}{|c|c|c|}
\hline Gene & Primer & Sequence $\left(5^{\prime} \rightarrow 3^{\prime}\right)$ \\
\hline \multirow[t]{2}{*}{ METTL3 } & Forward & CCAGCACAGCTTCAGCAGTTCC \\
\hline & Reverse & GCGTGGAGATGGCAAGACAGATG \\
\hline \multirow[t]{2}{*}{$E Z H 2$} & Forward & AATCAGAGTACATGCGACTGAGA \\
\hline & Reverse & GCTGTATCCTTCGCTGTTTCC \\
\hline \multirow[t]{2}{*}{ IL-6 } & Forward & CACATGTTCTCTGGGAAATCG \\
\hline & Reverse & TTGTATCTCTGGAAGTTTCAGATTGTT \\
\hline \multirow[t]{2}{*}{$M C P-1$} & Forward & СТСАССТGСТGСТАСТСАТTC \\
\hline & Reverse & GCATGAGGTGGTTGTGAAAAA \\
\hline \multirow[t]{2}{*}{$T N F-\alpha$} & Forward & GCCACCACGCTCTTCTGTCTAC \\
\hline & Reverse & GGGTCTGGGCCATAGAACTGAT \\
\hline \multirow[t]{2}{*}{ U6 } & Forward & TCCTCCACGACAACCAAAACC \\
\hline & Reverse & TCTTTTCCCAAAATCCCAGACTC \\
\hline \multirow[t]{2}{*}{ GAPDH } & Forward & CTGGGCTACACTGAGCACC \\
\hline & Reverse & AAGTGGTCGTTGAGGGCAATG \\
\hline
\end{tabular}


Table 1 Clinical data of patients.

\begin{tabular}{ll}
\hline $\begin{array}{l}\text { Clinicopathological characteristics of patients with } \\
\text { pneumonia }\end{array}$ \\
\hline Clinicopathological features (total = 50) \\
Gender \\
Male & 27 \\
Female & 23 \\
Median age (years) & 6 \\
Clinical manifestations & \\
Cough & 45 \\
Fever & 43 \\
Abnormal breathing & 30 \\
WBC (×109/L) & $10.50 \pm 3.40$ \\
Neutrophils $\times 109 / L)$ & $2.12 \pm 0.67$ \\
Path of infection & \\
Bacterial & 12 \\
Virus & 10 \\
Mycoplasma & 28 \\
\hline
\end{tabular}

Nanjing, China) was applied to amplify METTL3, EZH2, IL-6, MCP-1, and TNF- $\alpha$ complementary DNAs (CDNAs) using the QuantStudio 6 Flex real-time PCR (RT-PCR) system (Life Technologies, Carlsbad, CA, USA) according to product manual. The expression values of target gene were normalized to the U6 expression. Relative expressions of METTL3, $E Z H 2, I L-6, M C P-1$ and TNF- $\alpha$ were analyzed using the delta-delta $\mathrm{Ct}$ method $\left(2^{-\Delta \Delta C t}\right){ }^{16,17}$ Primer sequences are exhibited in Table 1. All reactions were executed in triplicate.

\section{Western blot assay}

Total protein was extracted from cells by radioimmunoprecipitation assay (RIPA) buffer (Beyotime, Shanghai, China). Bicinchoninic acid (BCA) protein assay kit (CoWin Biotechnology) was used to examine the total protein concentration. After separated by sodium dodecyl sulfatepolyacrylamide gel electrophoresis (SDS-PAGE), total protein was transferred to polyvinylidene difluoride membranes (PVDF; Millipore) and blocked for $1 \mathrm{~h}$ with $5 \%$ non-fat milk at room temperature. The protein was identified and incubated overnight at $4^{\circ} \mathrm{C}$ with specific primary antibodies METTL3 (ab195352, 1:1000; Abcam, Cambridge, MA, USA), EZH2 (ab191080, 1:500; Abcam), cleaved Caspase-3 (ab32042, 1:500; Abcam), cleaved Caspase-9 (ab2324, 1:2000; Abcam), JAK2 (ab108596, 1:1000; Abcam), p-JAK2 (ab32101, 1:5000; Abcam), STAT3 (ab31370, 1:1000; Abcam), p-STAT3 (ab76315, 1:5000; Abcam), and GAPDH (ab9485, 1:3,000; Abcam). Next, the membranes were incubated with horseradish peroxidase (HRP)-conjugated goat anti-rabbit immunoglobulin $\mathrm{G}$ secondary antibody (ab205718, 1:2000; Abcam), and the ECL chemiluminescence reagent (Beyotime) was added to obtain bands. GAPDH was used to normalize analyzed samples.

\section{Cell transfection}

Si-METTL3 and PcDNA-EZH2 vector (Genecreate, China) were used to knockdown or overexpress the expression of
METTL3 and EZH2. Cells were cultured in six-well plates for about $24 \mathrm{~h}$ and transfected with indicated plasmids using lipofectamine 3000 (Thermo Fisher Scientific, Grand Island, USA).

\section{Enzyme-linked-immunosorbent serologic assay (ELISA)}

IL-6, MCP-1, and TNF- $\alpha$ protein levels in WI-38 cells were examined by ELISA using kits purchased from eBioscience (Cat. No. 88-7013-88; San Diego, CA, USA) following manufacturer's instructions.

\section{MTT assay}

In order to evaluate the ability of cell proliferation, an MTT method was used. Briefly, the cells $\left(2.5 \times 10^{3}\right.$ cells $\left./ \mathrm{mL}\right)$ were plated into 96-well plates. After treatment, $0.5-\mathrm{mg} /$ $\mathrm{mL}$ MTT solution (Beyotime) was added into each well, and cells were incubated for another $4 \mathrm{~h}$ at $37^{\circ} \mathrm{C}$. Subsequently, the culture medium was discarded and $100-\mu \mathrm{L}$ dimethyl sulfoxide (DMSO, Sigma) was added for visualization.

\section{Cell Apoptosis}

Normal cultured cells were washed twice with phosphate buffer solution (PBS). Then $100-\mu \mathrm{L}$ binding buffer and $10-\mu \mathrm{L}$ FITC Annexin-V (20 $\mu \mathrm{g} / \mathrm{mL}$; Invitrogen) were added, and the cells were protected from light at room temperature for $30 \mathrm{~min}$. After this, the cells were added with $5-\mu \mathrm{L}$ PI (50 $\mu \mathrm{g} / \mathrm{mL}$; Invitrogen) and $400-\mu \mathrm{L}$ binding buffer. FACScan was used for flow cytometry quantitative detection (generally, for no more than $1 \mathrm{~h}$ ). Tube without FITC Annexin-V and PI was used as a negative control.

\section{Statistical analysis}

All data are presented as mean \pm standard deviation (SD) from three independent assays. Student's $t$-test was used to calculate comparisons between two groups. We used GraphPad Prism 5 (GraphPad Software, Inc., San Diego, CA, USA) for analysis. $\mathrm{P}<0.05$ was considered to manifest a statistically significant difference.

\section{Results}

\section{METTL3 and EZH2 were highly expressed in pediatric pneumonia patients and LPS-induced cell models}

The total RNA and protein were extracted from the peripheral blood mononuclear cells of 50 pediatric pneumonia patients and 50 healthy children of the same age to detect the expression levels of METTL3 and EZH2. The results of RT-qPCR and western blot analyses showed that both METTL3 and EZH2 were highly expressed in 50 pediatric pneumonia patients compared with 50 healthy subjects 


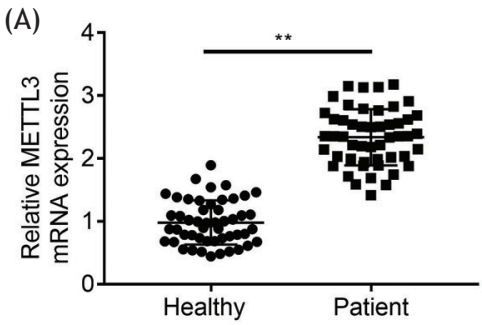

(C)

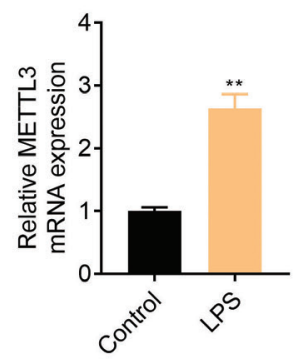

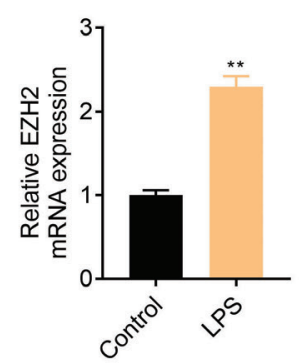

(D)

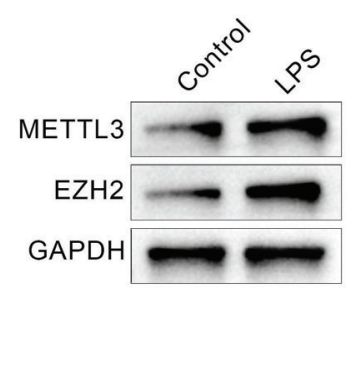

(B)

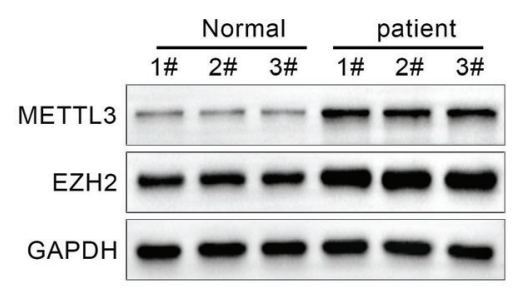

Figure 1 METTL3 and EZH2 were highly expressed in pediatric pneumonia patients and LPS-induced cell models. (A) mRNA and (B) protein expression levels of METTL3 and EZH2 in peripheral blood mononuclear cells (PBMC) obtained from pediatric pneumonia patients $(n=50)$ and even-aged healthy children (control group, $n=50$ ). (C) mRNA and (D) protein expression levels of METTL3 and EZH2 in WI-38 cells treated with LPS at a concentration of $20 \mu \mathrm{g} / \mathrm{mL}$, ${ }^{* *} \mathrm{P}<0.01$. GAPDH was normalized as an internal control.

(Figures $1 \mathrm{~A}$ and $1 \mathrm{~B})(\mathrm{P}<0.01)$. The expression levels of METTL3 and EZH2 in LPS-induced WI-38 cell model were detected. The results of RT-qPCR and western blot analyses suggested that the expression levels of METTL3 and EZH2 were notably upregulated in LPS-induced WI-38 cell model compared with the control group (Figures $1 \mathrm{C}$ and 1D) $(\mathrm{P}<0.01)$. These results illustrated that METTL3 and EZH2 were highly expressed in pediatric pneumonia patients and LPS-induced cell models.

\section{Downregulation of METTL3 abated LPS-induced inflammatory response}

We first detected transfection efficiency in LPS-induced WI-38 cells. Western blot assay revealed that the expression level of METTL3 declined in si-METTL3+LPS group (Figure $2 A)(P<0.01)$. The cellular inflammatory factors were examined by RT-qPCR assay and ELISA. The results showed that the expression levels and contents of IL-6, MCP-1, and TNF- $\alpha$ were significantly increased in LPS and si-NC+LPS groups, while the expression levels and contents of these inflammatory factors declined in si-METTL3+LPS group compared with the control group (Figures $2 B$ and $2 C)(P<0.01)$. The above-mentioned results validated that the knockdown of METTL3 suppressed LPS-induced inflammatory response.

\section{Downregulation of METTL3 inhibited LPS-induced apoptosis}

Next, we analyzed apoptosis in the indicated treated cells. MTT assay revealed that compared with the control group, cell viability was weakened in WI-38 cells induced with LPS whereas it was enhanced by knockdown of METTL3 (Figure 3A) $(P<0.01)$. Flow cytometry analysis suggested that LPS treatment increased whereas downregulating of METTL3 decreased the apoptosis rate of WI-38 cells (Figure $3 \mathrm{~B}$ ) $(P<0.01)$. Results of western blot assay demonstrated that compared with control group, the expression levels of cleaved caspase- 3 and cleaved caspase- 9 were increased in LPS and si-NC+LPS group whereas these were downregulated in si-METTL3+LPS group (Figure $3 C)(P<0.01)$. These results collectively demonstrated the suppression role of METTL3 in LPS-induced apoptosis.

\section{METTL3 regulated the JAK2-STAT3 signaling pathway through EZH2}

We further investigated the possible functional mechanism of METTL3 in pediatric pneumonia patients. According to the results of western blot assay, we found that inducing of LPS increased and overexpression of METTL3 decreased the expression level of EZH2 in WI-38 cells. The expression level of EZH2 in WI-38 cells was increased after transfecting with pcDNA EZH2 plasmid. Meanwhile, expression levels of p-JAK2-JAK2 and p-STAT3-STAT3 increased in LPS and si-NC+LPS group compared with the control group whereas overexpression of EZH2 reversed the expression reduction caused by knockdown of METTL3 (Figure 4) $(P<0.01)$. These results suggested that METTL3 regulated the JAK2STAT3 signaling pathway via EZH2.

\section{Downregulation of METTL3 inhibited inflammatory response and apoptosis through EZH2}

The contents of IL-6, MCP-1, and TNF- $\alpha$ were examined by ELISA. The results established that the contents of IL-6, MCP1 , and TNF- $\alpha$ decreased by different degrees in si-METTL3+pcDNA+LPS group compared with si-NC+pcDNA+LPS group 
(A)
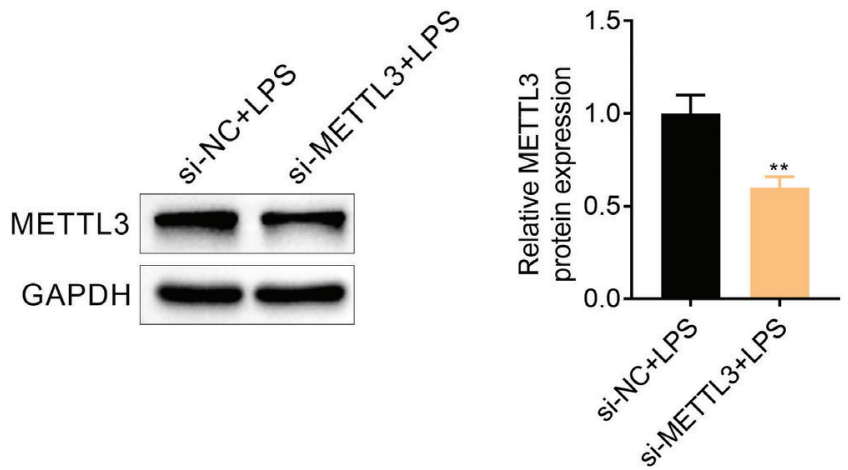

(B)

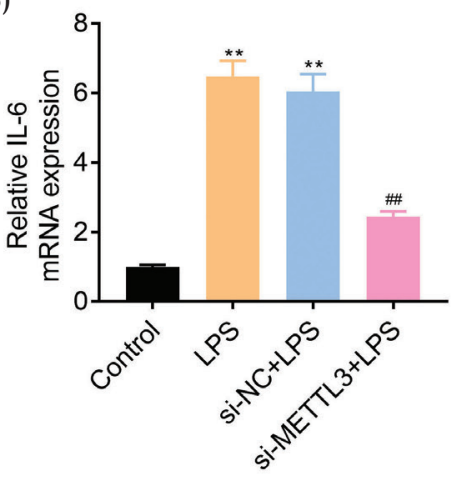

(C)

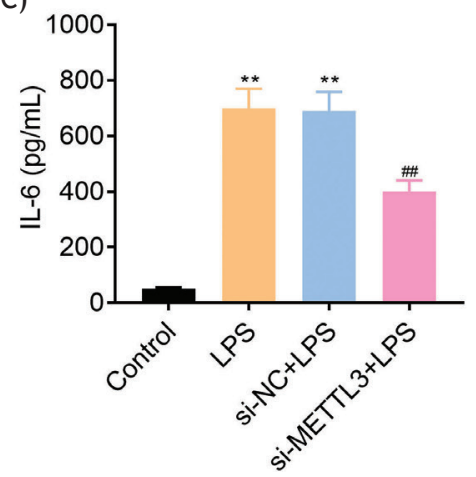

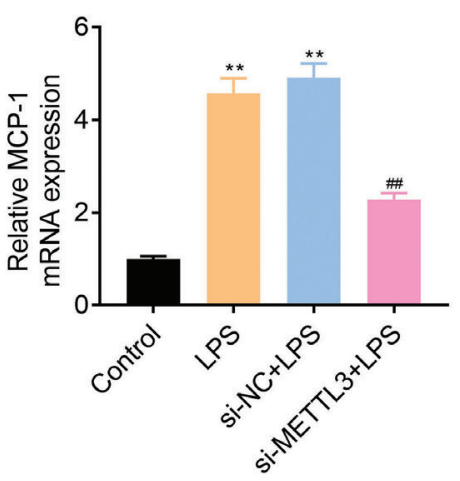
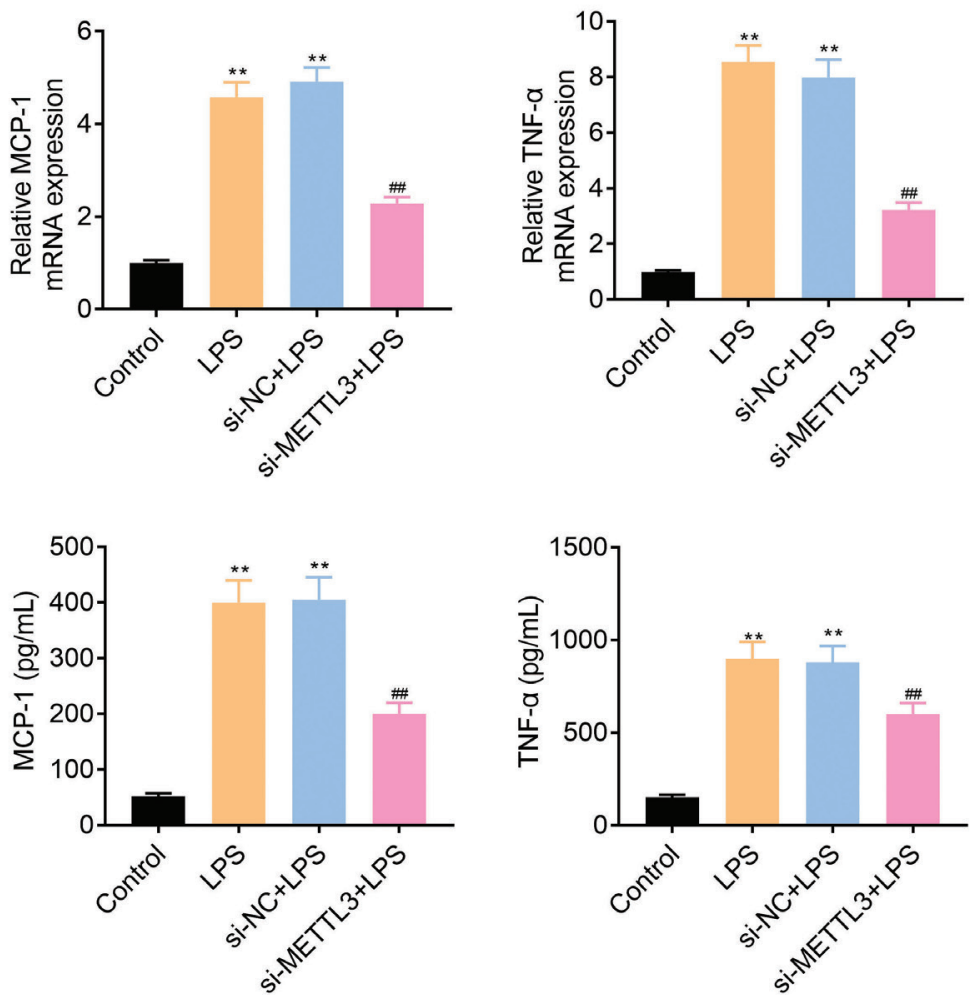

Figure 2 Downregulation of METTL3 abated LPS-induced inflammatory response. (A) The western blot assay results showed the protein expression level of METTL3 in LPS-induced WI-38 cells after transfecting with si-NC or si-METTL3 plasmids, ${ }^{* * P}<0.01$. GAPDH was normalized as an internal control. (B) RT-GPCR results exhibited the mRNA expression levels of IL-6, MCP-1, and TNF$\alpha$ in WI-38 cells of control, LPS, and si-NC+LPS and si-METTL3+LPS groups, ${ }^{* *} P<0.01$. (C) ELISA results showed the contents of IL-6, MCP-1, and TNF- $\alpha$ in WI-38 cells of control, LPS, and si-NC+LPS and si-METTL3+LPS groups, ${ }^{* * P}<0.01$. ${ }^{\#}$ Comparison between si-METTL3+LPS and si-NC+LPS groups.

whereas overexpression of EZH2 reversed the anti-inflammatory effect of si-METTL3 (Figure 5A) $(P<0.01)$. Results of western blot revealed that the expression levels of cleaved Caspase- 3 and cleaved Caspase- 9 decreased in si-METTL3+pCDNA+LPS group compared with si-NC+pCDNA+LPS group whereas overexpression of EZH2 reversed the apoptosis inhibited by si-METTL3 (Figure 5B) $(P<0.01)$. These two experiments validated that downregulation of METTL3 robustly suppressed inflammatory response and apoptosis via EZH2.

\section{Discussion}

Although the medical science in recent times has developed by leaps and bounds, the molecular mechanism of pediatric pneumonia is still unclear. Recently, some studies have reported that METTL3 is upregulated in a variety of inflammatory diseases. However, METTL3 is rarely studied in the case of pediatric pneumonia as its underlying regulatory mechanism is shrouded in a mist, and exploring this regulatory mechanism of METTL3 is particularly called for.

METTL3 proteins, which are highly expressed in tissues or organs and affect cell growth and apoptosis, have been validated to play a key role in regulating immunity and inflammation..$^{18}$ Disorders in METTL3 proteins play a vital role in the occurrence and progression of inflammation of cells or tissues..$^{19,20}$ Specifically, studies have also found that METTL3 proteins attenuate LPS-induced inflammatory response in macrophages by activating the NF- $\kappa B$ signaling pathway. ${ }^{6}$ Recently, the relationship has been established 
(A)

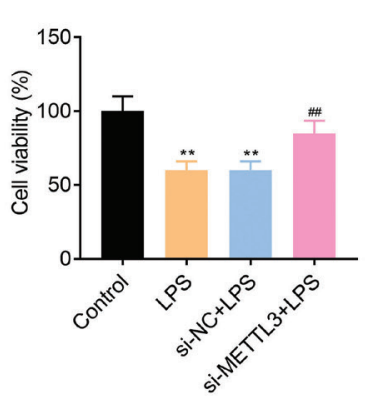

(C)

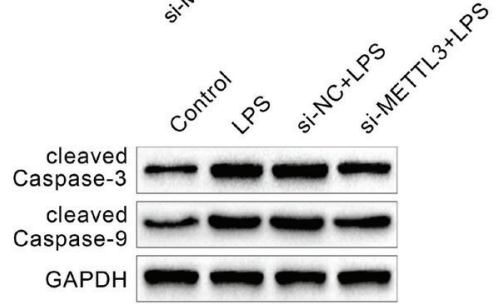

(B)

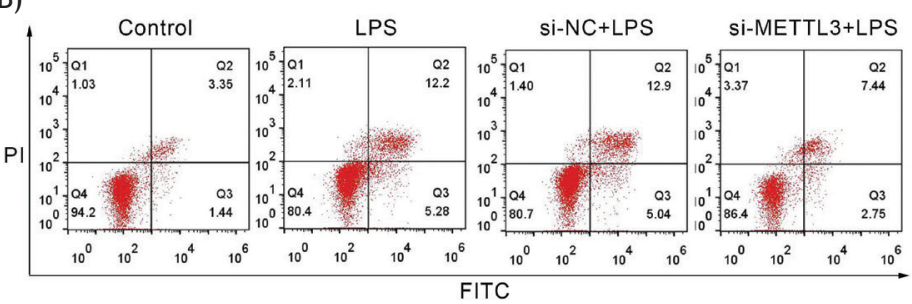

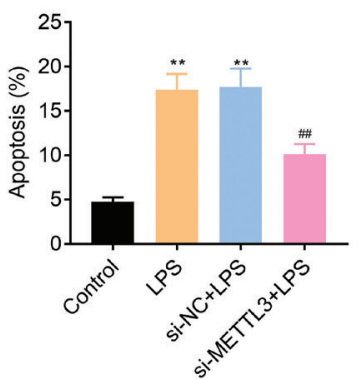
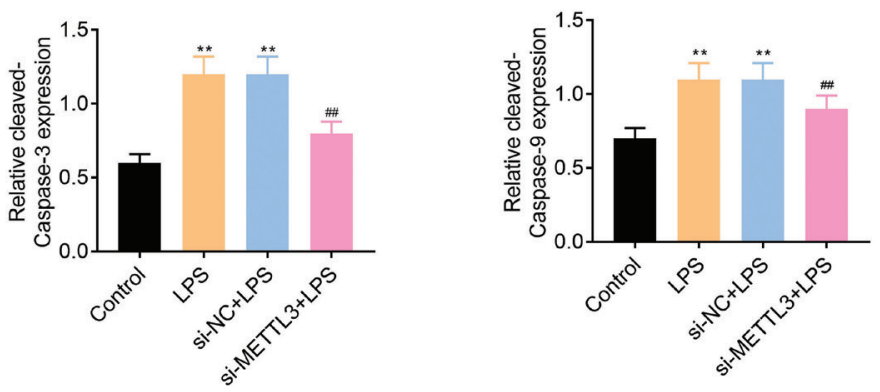

Figure 3 Downregulation of METTL3 inhibited LPS-induced apoptosis. (A) MTT and (B) flow cytometry assays detected cell viability and apoptosis rate in WI-38 cells of control, LPS, and si-NC+LPS and si-METTL3+LPS groups, ${ }^{* *}$ P $<0.01$. (C) Western blot analysis results exhibited the expression level of cleaved caspase- 3 and cleaved caspase- 9 in control, LPS, si-NC+LPS, and si-METTL3+LPS groups, ${ }^{* *} P<0.01$. GAPDH was normalized as an internal control. \#\#Comparison between si-METTL3+LPS and si-NC+LPS groups.
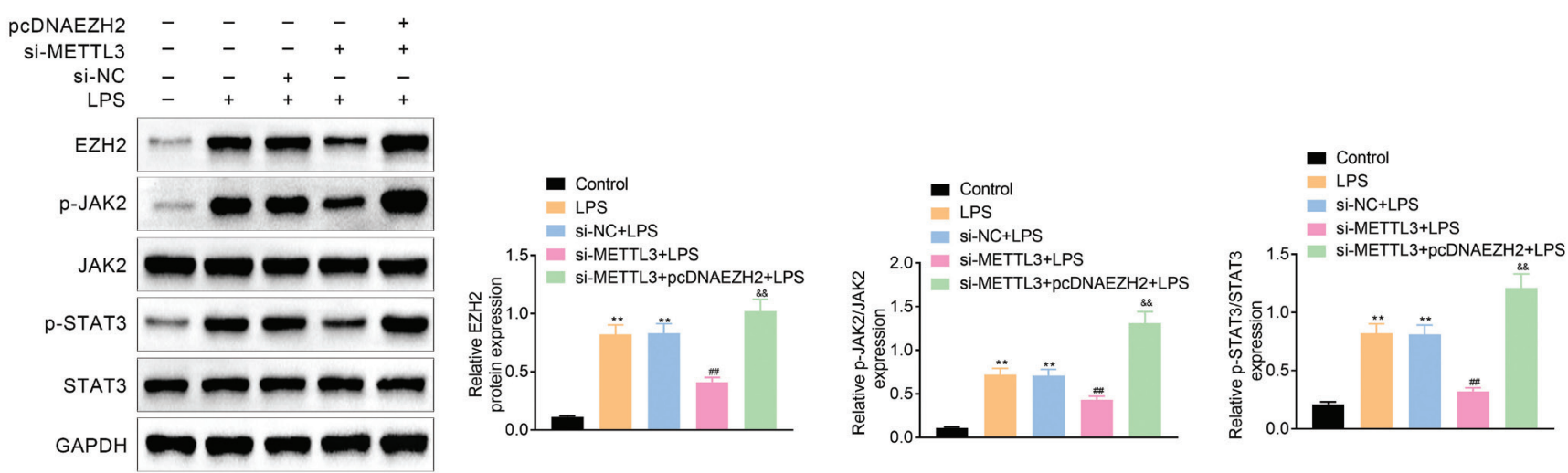

Figure 4 METTL3 regulated the JAK2-STAT3 signaling pathway via EZH2. The protein expression levels of EZH2, p-JAK2, JAK2, p-STAT3, and STAT3 in WI-38 cells of control, LPS, si-NC+LPS, si-METTL3+LPS, and si-METTL3+pcDNAEZH2+LPS groups, **P $<0.01$. GAPDH was normalized as an internal control. \#Comparison between si-METTL3+LPS and si-NC+LPS groups. Comparison between si-METTL3+pcDNAEZH2+LPS and si-METTL3+LPS groups.

between METTL3 and alternative splicing of MyD88 in LPS-induced inflammatory response in dental pulp cells. ${ }^{7}$ Moreover, METTL3 has been found to regulate immune responses to anti-PD-1 therapy. ${ }^{21}$ This study discloses that the mRNA and protein expression level of METTL3 is upregulated in peripheral blood monocytes from pediatric pneumonia patients and pediatric pneumonia cell model (WI-38). Suppression of METTL3 expression attenuated the inflammatory response and apoptosis in LPS-induced WI-38 cells. Hence, these results suggest that METTL3 functions as a regulatory factor in pediatric pneumonia.

METTL3 proteins execute their functioning by regulating the target mRNA's expression. ${ }^{22}$ Overexpression of
METTL3 promotes the progression of colorectal cancer by regulating miR-1246-SPRED2-MAPK signaling pathway. ${ }^{23}$ Moreover, METTL3 was reported to promote bladder cancer via AFF4-NF-אB-MYC network. ${ }^{24}$ In addition, METTL3 was reported to mediate osteoblast differentiation and inflammatory response through Smad and MAPK signaling pathway. ${ }^{25}$ Meanwhile, EZH2, JAK2, and STAT3 are targets of METTL3. EZH2 encodes a histone lysine $\mathrm{N}$-methyltransferase, and is involved in DNA methylation, thus inhibiting transcription of other genes. Mutation or overexpression of $\mathrm{EZH} 2$ has been associated with many cancer types. The protein encoded by the JAK2 gene is a non-receptor tyrosine kinase, a member of JAK family. The 
(A)
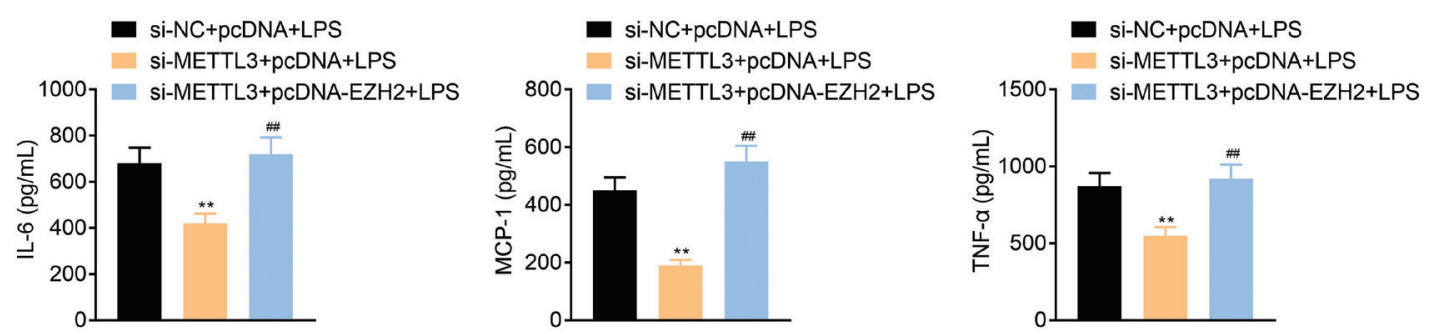

(B)
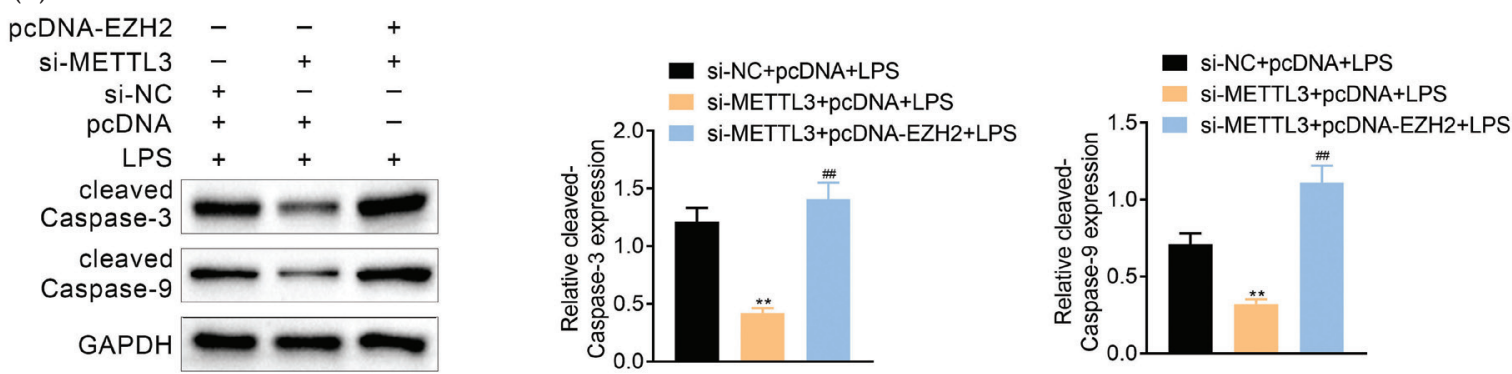

Figure 5 Downregulation of METTL3 inhibited inflammatory response and apoptosis through EZH2. (A) ELISA showed the contents of IL-6, MCP-1, and TNF- $\alpha$ in WI-38 cells of si-NC+pcDNA+LPS, si-METTL3+pcDNA+LPS, and si-METTL3+pcDNA-EZH2+LPS groups, ${ }^{* *} \mathrm{P}<0.01$. (B) Results of western blot analysis exhibited the expression level of cleaved caspase-3 and cleaved caspase-9 in WI-38 cells of si-NC+pcDNA+LPS, si-METTL3+pcDNA+LPS, and si-METTL3+pcDNA-EZH2+LPS groups, **P $<0.01$. GAPDH was normalized as an internal control. ${ }^{\#}$ Comparison between si-METTL3+LPS and si-NC+LPS groups.

protein encoded by SATA3 gene is a member of the STAT family of proteins regulating the expression of a variety of genes in response to cellular stimulation, thus playing a key role in many cellular processes such as cell growth and apoptosis. Knockdown of METTL3 in WI-38 cell lines would inhibit phosphorylation of JAK2 and SATA3 proteins whereas overexpression of EZH2 in WI-38 cell lines reversed the phosphorylation level of JAK2 and SATA3. Furthermore, the cell inflammatory response and apoptosis inhibited by knockdown of METTL3 were also reversed by the overexpression of EZH2, indicating that METTL3 promotes inflammation and cell apoptosis by regulating EZH2 in a pediatric pneumonia model.

In summary, it was established that METTL3 and EZH2 were overexpressed in peripheral blood monocytes of pediatric pneumonia patients or pediatric pneumonia cell model (WI-38). Moreover, suppression of METTL3 expression inhibited LPS-induced inflammatory response. Meanwhile, suppression of METTL3 expression also inhibited LPS-induced apoptosis. Finally, the knockdown of METTL3 in WI-38 cell lines would inhibit expression level of EZH2 and phosphorylation level of JAK2 and SATA3 proteins. Furthermore, the cell inflammatory response and apoptosis inhibited by the knockdown of METTL3 were reversed by the overexpression of EZH2. All these results explained the role of METTL3-EZH2 signaling pathway in promoting inflammation and cell apoptosis in a pediatric pneumonia model. This offers new ideas for the clinical therapy of pediatric pneumonia.

\section{Competing interests}

The authors had no conflicts of interest to disclose.

\section{Contribution of authors}

Ying Yang and Xiaoqing Yang designed the study and supervised data collection. Yi Wu analyzed and interpreted the data. Meijiao Fu prepared the manuscript for publication and reviewed manuscript's final draft. All the authors read and approved the final manuscript.

\section{References}

1. Hur I, Ozkan S, Halici A, Abatay K, Usul E, Cetin E, et al. Role of plasma presepsin, procalcitonin and c-reactive protein levels in determining the severity and mortality of community-acquired pneumonia in the emergency department. Signa Vitae. 2020;16:61-68. https://doi.org/10.22514/sv.2020.16.0034.

2. Zhang L, Dong L, Tang Y, Li M, Zhang M. MiR-146b protects against the inflammation injury in pediatric pneumonia through MyD88/NF- $\mathrm{KB}$ signaling pathway. Infect Dis (London). 2020;52(1):23-32. https://doi.org/10.1080/23744235.2019.16719 87

3. Piao X, Zou Y, Sui X, Liu B, Meng F, Li S, et al. Hydrostatin-SN10 ameliorates pancreatitis-induced lung injury by affecting IL-6induced JAK2/STAT3-associated inflammation and oxidative stress. Oxid Med Cell Longev. 2019;2019:9659757. https://doi. org/10.1155/2019/9659757

4. Li R, Hong $P$, Zheng $X$. $\beta$-carotene attenuates ipopolysaccharide-induced inflammation via inhibition of the NF-KB, JAK2/ STAT3 and JNK/p38 MAPK signaling pathways in macrophages. Anim Sci J (Nihon Chikusan Gakkaiho). 2019;90(1):140-8. https://doi.org/10.1111/asj.13108

5. Yu Y, Zhu T, Li Y, Jing L, Yang M, Li Y, et al. Repeated intravenous administration of silica nanoparticles induces pulmonary inflammation and collagen accumulation via JAK2/STAT3 and TGF- $\beta$ /Smad3 pathways in vivo. Int J Nanomed. 2019;14:723747. https://doi.org/10.2147/IJN.S209458 
6. Wang J, Yan S, Lu H, Wang S, Xu D. METTL3 attenuates LPSinduced inflammatory response in macrophages via NF- $\mathrm{KB}$ signaling pathway. Mediators Inflamm. 2019;2019:3120391. https://doi.org/10.1155/2019/3120391

7. Feng Z, Li Q, Meng R, Yi B, Xu Q. METTL3 regulates alternative splicing of MyD88 upon the lipopolysaccharideinduced inflammatory response in human dental pulp cells. J Cell Mol Med. 2018;22(5):2558-68. https://doi.org/10.1111/ jcmm.13491

8. Liu Q, Li M, Jiang L, Jiang R, Fu B. METTL3 promotes experimental osteoarthritis development by regulating inflammatory response and apoptosis in chondrocyte. Biochem Biophy Res Commun. 2019;516(1):22-7. https://doi.org/10.1016/j.bbrc. 2019.05.168

9. Wu R, Liu Y, Zhao Y, Bi Z, Yao Y, Liu Q, et al. m(6)A methylation controls pluripotency of porcine induced pluripotent stem cells by targeting SOCS3/JAK2/STAT3 pathway in a YTHDF1/ YTHDF2-orchestrated manner. Cell Death Dis. 2019;10(3):171. https://doi.org/10.1038/s41419-019-1417-4

10. Meng QZ, Cong CH, Li XJ, Zhu F, Zhao X, Chen FW. METTL3 promotes the progression of nasopharyngeal carcinoma through mediating M6A modification of EZH2. Eur Rev Med Pharm Sci. 2020;24(8):4328-36. https://doi.org/10.26355/eurrev_ 202004_21014

11. Liang $\mathrm{H}$, Huang $\mathrm{Q}$, Liao MJ, Xu F, Zhang $\mathrm{T}$, He J, et al. EZH2 plays a crucial role in ischemia/reperfusion-induced acute kidney injury by regulating p38 signaling. Inflamm Res Off J Eur Histamine Res Soc. 2019;68(4):325-36. https://doi. org/10.1007/s00011-019-01221-3

12. Hui $T$, Peng A, Zhao Y, Yang J, Ye L, Wang C. EZH2 regulates dental pulp inflammation by direct effect on inflammatory factors. Arch Oral Biol. 2018;85:16-22. https://doi. org/10.1016/j.archoralbio.2017.10.004

13. Lee S, Woo DC, Kang J, Ra M, Kim KH, Lee SR, et al. The role of the histone methyltransferase EZH2 in liver inflammation and fibrosis in STAM NASH mice. Biology. 2020;9(5):93. https:// doi.org/10.3390/biology9050093

14. Chen Z, Du Y, Liu X, Chen H, Weng X, Guo J, et al. EZH2 inhibition suppresses bladder cancer cell growth and metastasis via the JAK2/STAT3 signaling pathway. Oncol Lett. 2019;18(1):90715. https://doi.org/10.3892/ol.2019.10359
15. World Medical Association Declaration of Helsinki: ethical principles for medical research involving human subjects. J Amer Med Assoc (JAMA). 2013;310(20):2191-4. https://doi. org/10.1001/jama.2013.281053

16. Nolan T, Hands RE, Bustin SA. Quantification of mRNA using real-time RT-PCR. Nat Protocols. 2006;1(3):1559-82. https:// doi.org/10.1038/nprot.2006.236

17. Michaelidou K, Tzovaras A, Missitzis I, Ardavanis A, Scorilas A. The expression of the CEACAM19 gene, a novel member of the CEA family, is associated with breast cancer progression. Int J Oncol. 2013;42(5):1770-7. https://doi.org/10.3892/ijo.2013.1860

18. Liu S, Zhuo L, Wang J, Zhang Q, Li Q, Li G, et al. METTL3 plays multiple functions in biological processes. Amer J Cancer Res. 2020;10(6):1631-46. PMID: 32642280

19. Zeng C, Huang W, Li Y, Weng $\mathrm{H}$. Roles of METTL3 in cancer: mechanisms and therapeutic targeting. J Hematol Oncol. 2020;13(1):117. https://doi.org/10.1186/s13045-020-00951-w

20. Zhong $\mathrm{H}$, Hao L, Li X, Wang $\mathrm{C}$, Wu X. Anti-inflammatory role of trilobatin on lipopolysaccharide-induced acute lung injury through activation of AMPK/GSK3 $\beta$-Nrf2 pathway. Signa Vitae. 2020;16(2):160-6. https://doi.org/10.22514/sv.2020.16.0075

21. Wang $L$, Hui $H$, Agrawal $K$, Kang $Y$, Li N, Tang R, et al. $m(6)$ A RNA methyltransferases METTL3/14 regulate immune responses to anti-PD-1 therapy. EMBO J. 2020;39(20):e104514. https://doi.org/10.15252/embj.2020104514

22. Li B, Zhu J, Meng L. High expression of ACTL8 is poor prognosis and accelerates cell progression in head and neck squamous cell carcinoma. Mol Med Rep. 2019;19(2):877-84. https://doi. org/10.3892/mmr.2018.9716

23. Peng W, Li J, Chen R, Gu Q, Yang P, Qian W, et al. Upregulated METTL3 promotes metastasis of colorectal cancer via miR1246/SPRED2/MAPK signaling pathway. J Exp Clin Cancer Res. 2019;38(1):393. https://doi.org/10.1186/s13046-019-1408-4

24. Cheng $M$, Sheng L, Gao Q, Xiong Q, Zhang H, Wu M, et al. The m(6)A methyltransferase METTL3 promotes bladder cancer progression via AFF4/NF- $\mathrm{KB} / \mathrm{MYC}$ signaling network. Oncogene. 2019;38(19):3667-80. https://doi.org/10.1038/s41388-019-0683-z

25. Zhang Y, Gu X, Li D, Cai L, Xu Q. METTL3 regulates osteoblast differentiation and inflammatory response via smad signaling and MAPK signaling. Int J Mol Sci. 2019;21(1):199. https://doi. org/10.3390/ijms21010199 